High-Order Weno Simulations of

Three-Dimensional Reshocked

Richtmyer-Meshkov Instability to Late Times:

Dynamics, Dependence on Initial Conditions, and Comparisons to Experimental Data

O. Schilling, M. Latini

January 15, 2010

Acta Mathematica Scientia 
This document was prepared as an account of work sponsored by an agency of the United States government. Neither the United States government nor Lawrence Livermore National Security, LLC, nor any of their employees makes any warranty, expressed or implied, or assumes any legal liability or responsibility for the accuracy, completeness, or usefulness of any information, apparatus, product, or process disclosed, or represents that its use would not infringe privately owned rights. Reference herein to any specific commercial product, process, or service by trade name, trademark, manufacturer, or otherwise does not necessarily constitute or imply its endorsement, recommendation, or favoring by the United States government or Lawrence Livermore National Security, LLC. The views and opinions of authors expressed herein do not necessarily state or reflect those of the United States government or Lawrence Livermore National Security, LLC, and shall not be used for advertising or product endorsement purposes. 


\title{
HIGH-ORDER WENO SIMULATIONS OF THREE-DIMENSIONAL RESHOCKED RICHTMYER-MESHKOV INSTABILITY TO LATE TIMES: DYNAMICS, DEPENDENCE ON INITIAL CONDITIONS, AND COMPARISONS TO EXPERIMENTAL DATA
}

\author{
Oleg Schilling ${ }^{\mathrm{a}, *}$, Marco Latini ${ }^{\mathrm{b}, * *}$ \\ ${ }^{a}$ Lawrence Livermore National Laboratory, Livermore, California 94551 \\ ${ }^{b}$ Applied and Computational Mathematics, California Institute of Technology, Pasadena, California \\ 91125
}

\begin{abstract}
The dynamics of the reshocked multi-mode Richtmyer-Meshkov instability is investigated using $513 \times 257^{2}$ three-dimensional ninth-order weighted essentially nonoscillatory shock-capturing simulations. A two-mode initial perturbation with superposed random noise is used to model the Mach 1.5 air $/ \mathrm{SF}_{6}$ Vetter-Sturtevant shock tube experiment. The mass fraction and enstrophy isosurfaces, and density cross-sections are utilized to show the detailed flow structure before, during, and after reshock. It is shown that the mixing layer growth agrees well with the experimentally measured growth rate before and after reshock. The post-reshock growth rate is also in good agreement with the prediction of the Mikaelian model. A parametric study of the sensitivity of the layer growth to the choice of amplitudes of the short and long wavelength initial interfacial perturbation is also presented. Finally, the amplification effects of reshock are quantified using the evolution of the turbulent kinetic energy and turbulent enstrophy spectra, as well as the evolution of the baroclinic enstrophy production, buoyancy production, and shear production terms in the enstrophy and turbulent kinetic transport equations.
\end{abstract}

Keywords: Richtmyer-Meshkov instability, reshock, mixing properties, weighted essentially nonoscillatory (WENO) method

\section{Introduction}

The Richtmyer-Meshkov instability develops when perturbations on an interface separating gases with different properties grow following passage of a shock [3, 23]. Following the passage of the shock, the interface is accelerated along the direction of shock propagation and a transmitted shock enters the second gas. The misalignment of $\nabla \rho$ and $\nabla p$

\footnotetext{
Dedicated to Professor James G. Glimm on the occasion of his 75th Birthday

* Corresponding author

** Present address: CNA Corporation, Alexandria, VA, 22311

Email address: schilling1@llnl.gov (Oleg Schilling)
} 
deposits vorticity $\boldsymbol{\omega}=\boldsymbol{\nabla} \times \boldsymbol{u}$ on the interface through baroclinic vorticity production: in three dimensions

$$
\frac{\mathrm{d} \boldsymbol{\omega}}{\mathrm{d} t}=\frac{\boldsymbol{\nabla} \rho \times \boldsymbol{\nabla} p}{\rho^{2}}+\boldsymbol{\omega} \cdot \boldsymbol{\nabla} \boldsymbol{u}-\boldsymbol{\omega} \boldsymbol{\nabla} \cdot \boldsymbol{u},
$$

where $\mathrm{d} / \mathrm{d} t \equiv \partial / \partial t+\boldsymbol{u} \cdot \boldsymbol{\nabla}$ and viscous terms are neglected. The second term on the right side is zero in two-dimensional flows, in which there is no vortex stretching. The difference between two- and three-dimensional turbulence dynamics is largely associated with the physics of vortex stretching. The vorticity deposited on the interface by the shock drives the instability, resulting in interpenetrating bubble- and spike-like structures. Complex roll-ups of the evolving interface later appear. The transmitted shock reflects from the shock tube end wall and interacts with the evolving interface during reshock, depositing additional vorticity on the crenulated interface and generating additional small-scale structure within the mixing layer. As Richtmyer-Meshkov instability-induced flow is initiated by a shock wave, a direct numerical simulation (DNS) is not possible due to the prohibitively small scales needed to resolve the complex interactions of the shock with the density interfaces and the shocks themselves. As a result, numerical investigations of this instability have typically utilized conservative Eulerian shock capturing methods that do not resolve all of the spatial scales and small-scale interactions, but instead ensure that fundamental quantities are conserved across a shock and that the shock speed is accurately captured.

This study investigates various properties of the reshocked multimode RichtmyerMeshkov instability using three-dimensional $\left(513 \times 257^{2}\right)$ ninth-order weighted essentially nonoscillatory (WENO) simulations for the further development and eventual validation of turbulent transport and mixing models. The high-resolution, finite-difference, Eulerian, shock-capturing WENO method using a third-order total-variation diminishing (TVD) Runge-Kutta time-evolution scheme (see [1] and references therein) is applied here to a model of the Mach 1.5 air $/ \mathrm{SF}_{6}$ Vetter-Sturtevant Richtmyer-Meshkov instability shock tube experiment [27] for long evolution times. The membrane pushed through the wire mesh is modeled using a two-mode initial condition $[4,11]$, consisting of a high frequency and a low frequency component, together with superposed small-amplitude noise. The dynamics of reshock is explored using both isosurface visualizations and the time-evolution of the mixing layer width. A quantification of the sensitivity of the preand post-reshock mixing layer width on the choice of the initial amplitudes in the twomode initial perturbation is also presented. The numerical model of the experiment is validated by comparing to experimental amplitude data, and the simulations are extended to longer times than available in the experiment. The post-reshock simulation data is also compared to the prediction of the Mikaelian reshock model [19]. In addition, the amplification of turbulence within the mixing layer is investigated by considering the evolution of the turbulent kinetic energy and turbulent enstrophy spectra, as well as of important turbulent kinetic energy and enstrophy production mechanisms.

This paper is organized as follows. Previous experiments and simulations relevant to the present study are briefly summarized in Section 2. The numerical method, model of the initial interfacial perturbation, and the simulation of the reshocked RichtmyerMeshkov instability are summarized in Section 3, including a brief discussion of the benefits of formally high-order methods for investigating complex shocked flows. The dynamics of the instability evolution is illustrated through visualizations of the mass fraction and enstrophy isosurfaces, and density cross-sections in Section 4. The evolution 
of the mixing layer width is compared to the experimental data, and the sensitivity of the width to the choice of initial perturbation amplitudes are discussed in Section 5 . The amplification of the turbulence within the mixing layer by reshock is investigated using energy spectra and the principal production terms in the turbulent kinetic energy and enstrophy transport equations in Section 6. Finally, a discussion of the results and conclusions are presented in Section 7.

\section{Previous Experiments and Numerical Simulations}

Presented here is a brief review of the Vetter-Sturtevant experiments and previous numerical simulations and recent experiments relevant to the current investigation. In the subsequent description of previous and present simulations, it is assumed that the shock propagates along the $x$-direction.

\subsection{The Vetter-Sturtevant experiments}

Vetter and Sturtevant [27] performed shock tube experiments to investigate the effects of reshock of an evolving Richtmyer-Meshkov instability-induced mixing layer using a larger test-section than in previous experimental work. The larger test section allowed the growth of the mixing layer and evolution of the instability to be studied with minimal shock wave-boundary layer interaction. The $27 \mathrm{~cm} \times 27 \mathrm{~cm}$ square test section of the shock tube had a length of $61 \mathrm{~cm}$ for the 'short' experiments and $122 \mathrm{~cm}$ for the 'long' experiments, and was filled with air and sulfur hexafluoride $\left(\mathrm{SF}_{6}\right)$ gas separated by a thin nitrocellulose membrane supported by a wire mesh. The wire mesh had spacing of $1 \mathrm{~cm}$ and the initial perturbation had an amplitude of approximately $0.1 \mathrm{~cm}$. A shock wave with a Mach number ranging from $M a=1.18$ to 1.98 was launched in the air, ruptured the membrane, and refracted at the interface. The transmitted shock then reflected from the shock tube end wall and reshocked the evolving interface. High-resolution sparkSchlieren images were obtained to study flow features and obtain measurements of the growth of the mixing layer. The experimental configuration allowed the measurement of the width $h$ of the mixing layer both after the initial shock passage and through the reshock phase. Additional details of the experiments are available elsewhere [11, 17].

The measured growth rates obtained from the experiments were compared to the analytical prediction of Mikaelian [19] for the shock-induced reacceleration of an evolving interface

$$
h(t)=0.28 \Delta u_{1} A^{+} t,
$$

where $\Delta u_{1}$ is the change in the interface velocity caused by the second shock, $A^{+}$is the post-reshock Atwood number, and $t$ is time. This phenomenological model is based on results from the rocket-rig experiments of Read [21] and Youngs [29] for the growth of Rayleigh-Taylor unstable mixing layers; the empirical coefficient 0.28 is based on the mixing layer growth rate from the experiments [21]. Note that (2) is independent of the initial perturbation amplitude and predicts a linear-in-time growth of the mixing layer width. The measured values in the experiment were one order of magnitude lower than the analytical predictions. Better agreement was obtained when the grid was placed downstream from the interface: in this configuration the shock pushed the interface through the wire mesh causing it to fragment. In this case, the results were approximately one third smaller than the analytical predictions, suggesting that the membrane 
fragmentation may have significantly damped the mixing. Agreement with the Mikaelian model after reshock was observed for all cases, indicating that the structure following reshock is weakly dependent on the initial conditions.

\subsection{The Leinov et al. experiments}

More recently, the effect of reshock on Richtmyer-Meshkov mixing arising from different arrival times of the reflected shock at the evolving mixing layer (achieved by changing the test section length from $80 \mathrm{~cm}$ to $172 \mathrm{~cm}$ ) and from light-to-heavy and heavy-to-light gas configurations was studied using Schlieren imaging in $M a=1.15-1.45$ air $/ \mathrm{SF}_{6}$ experiments $[16,17]$. A thin membrane separated the gases and had randomly distributed small-scale perturbations with random wavelengths ranging up to $\lambda \sim 0.1 \mathrm{~cm}$. The evolution of the mixing layer following reshock was found to be independent of its amplitude at the time of reshock, but dependent on the strength of the shock reflected from the end wall. The mixing layer grew linearly in time following reshock and before the interaction of the reflected rarefaction with the evolving interface. The growth rate was independent of the reshock strength when normalized by the distance traveled. The results were in good agreement with the Mikaelian model (2), but with coefficients ranging from 0.28 to 0.39 from several sets of experiments. A comparison of the experimental results to those from arbitrary Lagrangian-Eulerian (ALE) simulations using the LEEOR3D code suggested that the post-reshock linear growth is a result of bubble competition.

\subsection{Simplified piecewise-parabolic method simulations}

Cohen et al. [4] performed a highly resolved $\left(2048^{2} \times 1920\right)$ three-dimensional simplified piecewise-parabolic method (SPPM) simulation of the $M a=1.5$ Vetter and Sturtevant experiment before reshock with the wire mesh downstream of the interface. To approximate the thin membrane supported by a wire mesh, a two-mode perturbation of the form

$$
\eta(y, z)=0.01[|\sin (k y)||\sin (k z)|-\cos (2 \pi y) \cos (2 \pi z)],
$$

was chosen, where $k=10 \pi$. The first term represents the small-scale perturbation associated with the wire mesh, and the second term represents the scale associated with the transverse dimension of the shock tube scaled to have a width of $2 \pi$. The study of the evolution and interaction of a two-scale initial perturbation provides a simple model for the fundamental pairwise interaction of scales in turbulence. The simulation was performed using a simplified form of the piecewise-parabolic method (PPM) [5] applied to the Euler equations for compressible ideal gases. The single-fluid limitation of the code used was not expected to affect the macroscopic behavior of the flow for relatively weak $M a=1.5$ shocks; the adiabatic exponent was chosen as $\gamma=1.3$.

The flow was visualized by considering the entropy $s=\ln \left(p / \rho^{\gamma}\right)$ as it well tracks the contact discontinuity and exhibits a small change across the shock. The mixing layer width, $h(t)$, was defined in terms of the entropy as follows. Let $s_{1}$ and $s_{2}$ be the value of the entropy to the left and to the right of the mixing layer, respectively. Let $\bar{s}=\left(s_{1}+s_{2}\right) / 2$. Define $x_{1}$ as the location where transverse slices have range $\left[s_{1}, \bar{s}\right]$ and $x_{2}$ as the location where the range is $\left[\bar{s}, s_{2}\right]$. Then the mixing layer width is the difference $h=x_{2}-x_{1}$. The mixing layer width obtained from the simulation and from the experiment were in generally good agreement. 


\subsection{Large-eddy simulations}

Hill, Pantano and Pullin [11] performed large-eddy simulations (LES) of the Vetter and Sturtevant experiments using a hybrid tuned center-difference (TCD) and fifth-order WENO scheme, and the stretched vortex subgrid-scale model. A third-order strongstability preserving Runge-Kutta time-evolution scheme was used. The initial perturbation was of the form

$$
\eta(y, z)=a_{0}\left|\sin \left(\frac{\pi y}{\lambda}\right) \sin \left(\frac{\pi z}{\lambda}\right)\right|+b_{0} \psi(y, z),
$$

where $\lambda \approx 27 \mathrm{~cm} / 14 \approx 2 \mathrm{~cm}, a_{0}=0.25 \mathrm{~cm}, b_{0}=0.025 \mathrm{~cm}$, and $\psi(y, z)$ is a noise with prescribed spectrum $k^{4} \exp \left[-\left(k / k_{0}\right)^{2}\right]$ with $k_{0}=4$ corresponding to a peak wavelength $\pi / \sqrt{8} \mathrm{~m}$. Simulations were performed for $M a=1.24,1.5$, and 1.98. The grid resolutions were $616 \times 128^{2}$ and $327 \times 128^{2}$ for the smallest and largest Mach numbers, respectively, and $388 \times 128^{2}$ and $776 \times 256^{2}$ for the two intermediate Mach number simulations. Binary mixture relations were used to compute transport coefficients and thermodynamic quantities. The growth rates from the LES were is good agreement with those measured from the experiments.

More recently, Ukai et al. [26] performed three-dimensional $746 \times 140^{2}$ LES of the $M a=1.5$ Vetter-Sturtevant experiment using a one-equation model for the subgridscale kinetic energy. A hybrid finite-volume method was used, in which a fourth-order central scheme is used in smooth flow regions. The flux evaluation used a high-order flux difference splitting method in regions with strong gradients. A second-order predictorcorrector time-evolution scheme was used. The initial condition (4) was also used. While the mixing growth rate before reshock was overestimated, the growth rate following reshock was in good agreement with the experimental data. Vortical structures were visualized by using isosurfaces of the Q-criteria, and two regimes with strong vorticity were identified at the edges of the mixing layer.

\section{Equations Solved and the Numerical Method}

The WENO reconstruction used in the present study is briefly described, and its benefits for investigating complex hydrodynamic flows with shocks are discussed. Formally higher order reconstructions are less numerically dissipative and have greater resolving power than lower order reconstructions [14, 30]. Thus, high-order WENO methods are suitable for investigating multidimensional shock-driven flows in which the dynamics of a wide range of spatial scales and complex wave structures must be characterized with high fidelity.

\subsection{Equations solved and description of the WENO algorithm}

Simulations were performed using the characteristics-based finite-difference WENO method $[1,25]$. The Euler equations were augmented by the mass fraction to track the 
mixing dynamics

$$
\frac{\partial}{\partial t}\left[\begin{array}{c}
\rho \\
\rho u \\
\rho v \\
\rho w \\
\rho e \\
\rho m
\end{array}\right]+\frac{\partial}{\partial x}\left[\begin{array}{c}
\rho u \\
\rho u^{2}+p \\
\rho u v \\
\rho u w \\
(\rho e+p) u \\
\rho m u
\end{array}\right]+\frac{\partial}{\partial y}\left[\begin{array}{c}
\rho v \\
\rho u v \\
\rho v^{2}+p \\
\rho v w \\
(\rho e+p) v \\
\rho m v
\end{array}\right]+\frac{\partial}{\partial z}\left[\begin{array}{c}
\rho w \\
\rho u w \\
\rho v w \\
\rho w^{2}+p \\
(\rho e+p) w \\
\rho m w
\end{array}\right]=0
$$

where $\rho$ is the density, $\mathbf{u}=(u, v, w)$ is the velocity, $p=\rho R T$ is the ideal gas pressure $(R$ is the gas constant), $e=\left(u^{2}+v^{2}+w^{2}\right) / 2+p /(\gamma-1)$ is the total energy per unit mass, and $m$ is the mass fraction of the denser gas. A convex nonlinearly-weighted combination of all polynomial flux reconstructions is used to achieve the essentially nonoscillatory property and formally high-order accuracy in smooth flow regions. Ninth-order reconstruction was used for its desirable properties of reduced numerical dissipation and better preservation of small-scale structures [14].

The eigensystem of fluxes in the Euler equations is obtained from the Jacobian of the Roe-averaged fluxes in each spatial direction. The linearized Roe Riemann solver [22] is used to obtain the eigenvalues and eigenvectors. Lax-Friedrichs flux-splitting is used to split the original fluxes into their positive and negative components. The positive and negative flux components are then projected in the characteristic fields using the left eigenvectors to form the positive and negative characteristic fields at each cell center. An rth-degree piecewise-polynomial is then used to reconstruct the projected fluxes at the cell boundaries with high-order accuracy: a weighted convex combination of all possible $r t h$-degree piecewise-polynomial approximations ( $r=5$ for ninth-order) of the characteristic fields using the neighboring cell-centered values is constructed and evaluated at the boundaries of a given cell. For a given reconstruction order, there are $r$ possible $r$ th-degree piecewise-polynomials, with properties depending on the smoothness of the underlying solution. As the polynomials may use stencils containing discontinuities (and, thus, induce Gibbs oscillations), a weighted average of all of the possible polynomial reconstructions at a point is computed. The weights of the $r$ possible stencils around a given cell center are computed from the projected flux via a divided difference. Essentially zero weights are assigned to polynomials crossing discontinuities and nearly-equal weights are assigned to polynomials over smooth regions. The formal order of accuracy for the derivative of the flux is $2 r-1$ in smooth flow regions. The semi-discrete equations are evolved in time using the third-order total variation diminishing (TVD) Runge-Kutta method [1].

Conservative finite-difference discretizations of the Euler equations with WENO flux reconstruction contain implicit truncation errors that can be regarded as a nonlinear, adaptive numerical dissipation. The present simulations can thus be interpreted as a class of implicit large-eddy simulations (ILES) [2, 8-10], in which the equations are implicitly filtered by the discretization and the numerical dissipation is a surrogate for an explicit subgrid-scale model. As the nondissipative compressible fluid dynamics equations are formally ill-posed, this numerical dissipation regularizes the numerical method. As a result, quantities obtained from such simulations depend on the resolution and cannot be regarded as fully resolved. ILES methods typically dissipate velocity and scalar fluctuations approximately in the same manner numerically: the numerical Schmidt number is of $O(1)$, which may provide a reasonable model for the mixing of ideal gases. The 
reduced numerical dissipation of WENO schemes (or modifications thereof) can be coupled with explicit subgrid-scale models to perform conventional large-eddy simulations (LES) [11].

\subsection{Benefits of the WENO method for simulating complex shocked flows}

Formally high-order methods better resolve complex flow features at long evolution times than second- and third-order methods. Higher order methods are also more computationally efficient than lower order methods for the same accuracy [12, 13]. In particular, sufficiently high order WENO methods are well-suited for simulating complex, compressible evolving flows containing shocks and structures containing a wide range of scales. For example, the advantage of WENO methods is realized in complex multidimensional reshocked Richtmyer-Meshkov unstable flows [14]. Upwinding in the WENO method leads to large numerical dissipation in relatively smooth flow regions away from shocks. Methods for reducing this dissipation by hybridizing the WENO method with a highorder scheme, and for improving the resolving power are discussed elsewhere [14]. In the present work, higher-order (ninth-order) WENO reconstruction is used instead to achieve lower numerical dissipation.

\section{Three-Dimensional Simulations of Reshocked Richtmyer-Meshkov Insta- bility}

Numerical simulations were performed using the ninth-order WENO method. Presented here are the numerical simulation parameters, initial and boundary conditions, and grid resolution. Mass fraction isosurfaces are used to visualize the instability evolution following the passage of the shock, during reshock, and at late times following reshock. In addition, enstrophy isosurfaces and cross-sections of the density within the mixing layer are presented to visualize the small-scale structures as the instability develops spatially and temporally.

\subsection{Numerical simulation parameters}

The numerical simulation parameters were chosen to closely correspond to the $M a=$ 1.5 shock tube experiment of Vetter and Sturtevant [27]. The shock tube had a test section of length $61 \mathrm{~cm}$ with a square cross section of size $27 \mathrm{~cm}$. The initial gases are air and sulfur hexafluoride $\mathrm{SF}_{6}$, with the shock initially launched in air so that the initial refraction is from a lighter gas into a heavier gas. The initial temperature and pressure were $T_{1}=296{ }^{\circ} \mathrm{K}$ and $p_{1}=1$ bar, respectively.

\subsubsection{Initial gas composition}

In the experiments, a membrane supported by a wire mesh located at the entrance of the test section initially separated the air and $\mathrm{SF}_{6}$ gases. In the present simulations the properties of air and $\mathrm{SF}_{6}$ gases are used to determine the properties of a mixture assumed to be composed of $50 \%$ air and $50 \% \mathrm{SF}_{6}$ by volume, denoted $\operatorname{air}\left(\mathrm{SF}_{6}\right)$. The properties of the gases are given in Table 4.1.1, and the properties of the mixture are determined as follows. 


\begin{tabular}{|c||c|c|c||}
\hline & air & $\mathrm{SF}_{6}$ & $\operatorname{air}\left(\mathrm{SF}_{6}\right)$ \\
\hline \hline$M(\mathrm{~g} / \mathrm{mol})$ & 28.95 & 146.05 & 84.62 \\
$\rho\left(\mathrm{g} / \mathrm{cm}^{3}\right)$ & $1.202 \times 10^{-3}$ & $5.494 \times 10^{-3}$ & $3.348 \times 10^{-3}$ \\
$\gamma$ & 1.4 & 1.093 & 1.1557 \\
$R(\mathrm{erg} / \mathrm{g} \mathrm{K})$ & $2.872011 \times 10^{6}$ & $5.692894 \times 10^{5}$ & $9.826513 \times 10^{5}$ \\
$c_{p}(\mathrm{erg} / \mathrm{g} \mathrm{K})$ & $1.005204 \times 10^{7}$ & $6.690681 \times 10^{6}$ & $7.294079 \times 10^{6}$ \\
$c_{v}(\mathrm{erg} / \mathrm{g} \mathrm{K})$ & $7.180028 \times 10^{8}$ & $6.121392 \times 10^{6}$ & $6.311427 \times 10^{6}$ \\
\hline
\end{tabular}

Table 1: Properties of air, $\mathrm{SF}_{6}$, and air $\left(\mathrm{SF}_{6}\right)$ mixture used in the WENO simulations.

The mixture density is $\rho_{a i r\left(S F_{6}\right)}=\left(\rho_{a i r}+\rho_{S F_{6}}\right) / 2$ and the mass fraction of air in the mixture is $m_{\text {air }}=\rho_{\text {air }} /\left(2 \rho_{\text {air }\left(S F_{6}\right)}\right)$. This mass fraction is then used to determine the new adiabatic exponent of the mixture according to [20]

$$
\gamma_{a i r\left(S F_{6}\right)}=\frac{m_{a i r} c_{p, a i r}+\left(1-m_{a i r}\right) c_{p, S F_{6}}}{m_{a i r} c_{v, a i r}+\left(1-m_{a i r}\right) c_{v, S F_{6}}} .
$$

From this new value of the adiabatic exponent, standard ideal gas relations are used to determine new values for the heat capacities for both air and $\mathrm{SF}_{6}, c_{p}$ and $c_{v}$, and the molecular weight of the mixture (see Table 4.1.1). This procedure gives $\gamma_{a i r\left(S F_{6}\right)}=1.557$ used as the adiabatic exponent for both the air and $\mathrm{SF}_{6}$ gases in the simulations. As the ratio of specific heats is constant for both gases in this study, some properties of the flow may not be predicted very accurately (i.e., transmitted and reflected shock speeds, time of reshock, initial interface velocity), but the mixing properties are not expected to be strongly affected by the single- $\gamma$ formulation, as the flow is nearly incompressible over most of the evolution for the Mach number considered [11, 18]. Selecting the shock Mach number to match the initial velocity of the gases leads to a better match with the experimental reshock condition [14]. As a result, the shock Mach number is adjusted to match the initial growth rate predicted by linear stability analysis.

\subsubsection{Computational domain, grid resolution, and boundary conditions}

To match the shock tube test section dimension, the computational domain $\left[0, L_{x}\right] \times$ $\left[0, L_{y}\right] \times\left[0, L_{z}\right]$ has spanwise dimension of $L_{y}=L_{z}=27 \mathrm{~cm}$, with the perturbed initial interface located $3 \mathrm{~cm}$ from the edge of the shock tube. Thus, to obtain a test section of length $L_{x}=61 \mathrm{~cm}$, the total length of the computational domain along the streamwise direction is $L_{x}=64 \mathrm{~cm}$, as illustrated in Fig. 1. The small-wavelength perturbation corresponding to the membrane pushed through the wire mesh is clearly visible with 10 wavelengths per transverse direction. The grid resolution was $513 \times 257^{2}$.

The following boundary conditions were used: (1) inflow at the test section entrance in the streamwise $(x)$ direction; (2) reflecting at the end wall of the test section in the streamwise direction, and; (3) periodic in the spanwise ( $y$ and $z$ ) directions corresponding to the cross-section of the test section. The reflecting boundary condition is implemented by reversing the normal component of the velocity vector: $u(\boldsymbol{x}, t)=-u(\boldsymbol{x}, t)$ at $x=L_{x}$ and at the ghost points, which is exact and does not generate spurious noise. 


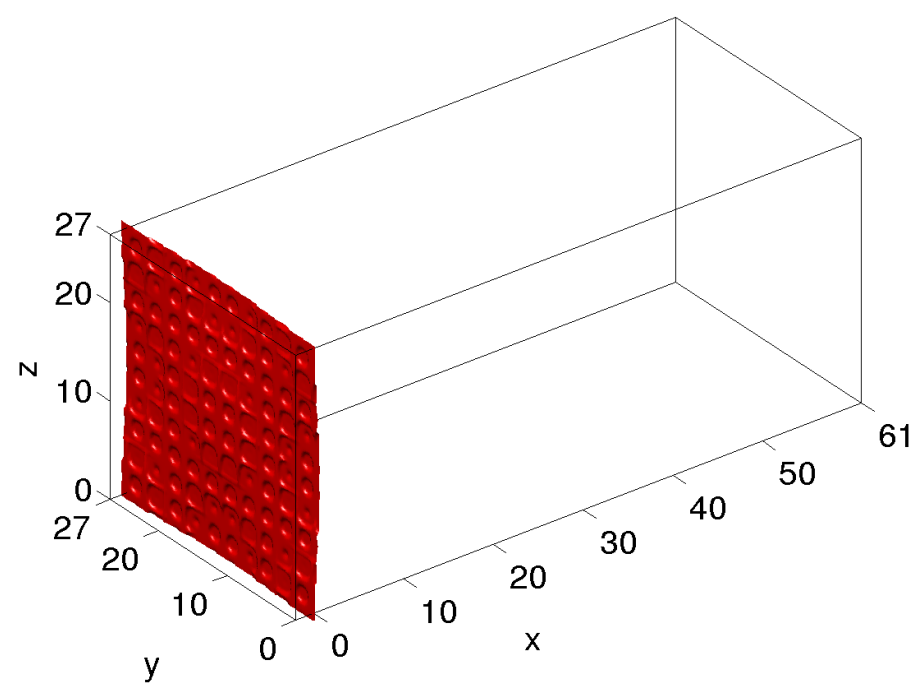

Figure 1: Illustration of the shock tube test section having a length of $61 \mathrm{~cm}$ and square cross-sectional width of $27 \mathrm{~cm}$ considered in the computational domain.

\subsection{Model of the initial interfacial perturbation}

A two-mode initial perturbation with random noise was used to model the shock tube experiment. The initial conditions generalize the two-mode model of Cohen et al. (3) with random noise similar to that of Hill et al. [11],

$$
\eta(y, z)=a\left|\sin \left(k_{1 y} y\right) \sin \left(k_{1 z} z\right)\right|-b \cos \left(k_{2 y} y\right) \cos \left(k_{2 z} z\right)+\psi(y, z),
$$

where $a$ and $b$ are the initial perturbation amplitudes, $\psi(y, z)$ is a small-scale stochastic perturbation, and the initial perturbation wavenumbers are

$$
k_{1 y}=\frac{2 \pi n_{1 y}}{\lambda_{y}}, k_{1 z}=\frac{2 \pi n_{1 z}}{\lambda_{z}}, k_{2 y}=\frac{2 \pi n_{2 y}}{\lambda_{y}}, k_{2 z}=\frac{2 \pi n_{2 z}}{\lambda_{z}} .
$$

In the present simulations $n_{1 y}=n_{1 z}=5$ and $n_{2 y}=n_{2 z}=1$ are the initial perturbation mode numbers, and $\lambda_{y}=\lambda_{z}=27 \mathrm{~cm}$ are the initial perturbation wavelengths, so that

$$
\eta(y, z)=a\left|\sin \left(\frac{10 \pi y}{27}\right) \sin \left(\frac{10 \pi z}{27}\right)\right|-b \cos \left(\frac{2 \pi y}{27}\right) \cos \left(\frac{2 \pi z}{27}\right)+\psi(y, z) .
$$

The first term models the membrane pushed through the wire mesh, the second term models the mesh distortion, and the stochastic noise $\psi(y, z)$ models the fragmentation of the membrane. The superimposed noise on the deterministic two-mode perturbation breaks symmetry and accelerates the development of nonlinear modal interactions. The pre-shock Atwood number is $A^{-}=0.641$ and the initial amplitudes are $a=0.0675$ $\mathrm{cm}$ and $b=0.00675 \mathrm{~cm}\left(0.25 \%\right.$ and $0.025 \%$ of $L_{z}$, respectively). The smallest values supported on a $513 \times 257^{2}$ grid are $a=0.05 \mathrm{~cm}$ and $b=0.005 \mathrm{~cm}$. 


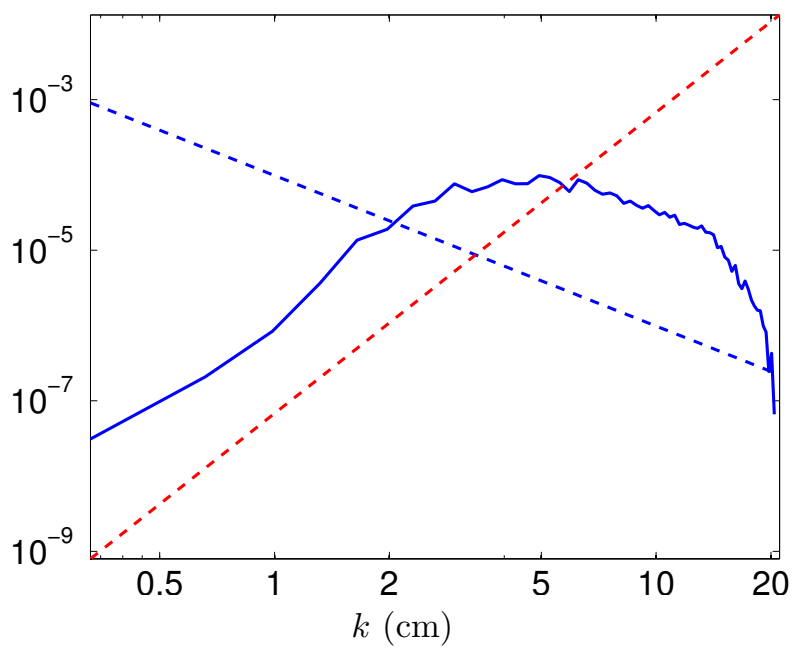

Figure 2: Spectrum $E_{\psi}(k)$ of the small-scale stochastic perturbation $\psi(x, y)$ used to break the symmetry, shown on a log-log scale. Fiducials are shown for $k^{4}$ (red dashed line) and $k^{-2}$ (blue dashed line).

The spectrum of the Fourier transform of the initial small-scale stochastic noise perturbation $\widehat{\psi}(k)$ is taken to be

$$
E_{\psi}(k)=|\widehat{\psi}(k)|^{2}=k^{-2}\left[\frac{k L}{\sqrt{(k L)^{2}+B}}\right]^{4},
$$

where $k=\sqrt{k_{y}^{2}+k_{z}^{2}}$ is the two-dimensional wavenumber, $L$ is a lengthscale, and $B$ is a dimensionless free parameter. This spectrum is an adaptation of the von Kármán spectrum [28] with the following properties:

1. for small $k L$, the spectrum grows as $E_{\psi}(k) \sim k^{4}$;

2. for large $k$, the spectrum scales as $E_{\psi}(k) \sim k^{-2}$, approximating the surface perturbations observed in many physical situations including the deuterium-tritium ice surface roughness in inertial confinement fusion (ICF) target capsules [7].

The values $L=0.95 \mathrm{~cm}$ and $B=5 \sqrt{2}$ are adopted in the present interfacial perturbation model, corresponding to a peak in the spectrum that matches the dimension of the wire mesh in the experiment: the spectrum $E_{\psi}(k)$ is shown in Fig. 2. Hill et al. [11] and Ukai et al. [26] instead assumed a Gaussian spectrum for the noise. The initial condition (3) is a special case of the present initial condition corresponding to $a=b=0.27 \mathrm{~cm}$ and $\psi=0$. As a thin membrane initially separated the two gases in the experiments, the modeled initial interface in the present WENO simulations is sharp (nondiffusive).

The stochastic noise $\psi(y, z)$ is created in wavenumber space by constructing the Fourier transform $\widehat{\psi}\left(k_{y}, k_{z}\right)$ as follows. Let $\Delta k_{y}=2 \pi / L_{y}$ and $\Delta k_{z}=2 \pi / L_{z}$ and use these to define the wavenumbers $k_{y}$ and $k_{z}$ corresponding to a $N_{y} \times N_{z}$ matrix in $k$ space. Next, determine the vector magnitude for each of the elements in the matrix 
$\psi(y, z)$
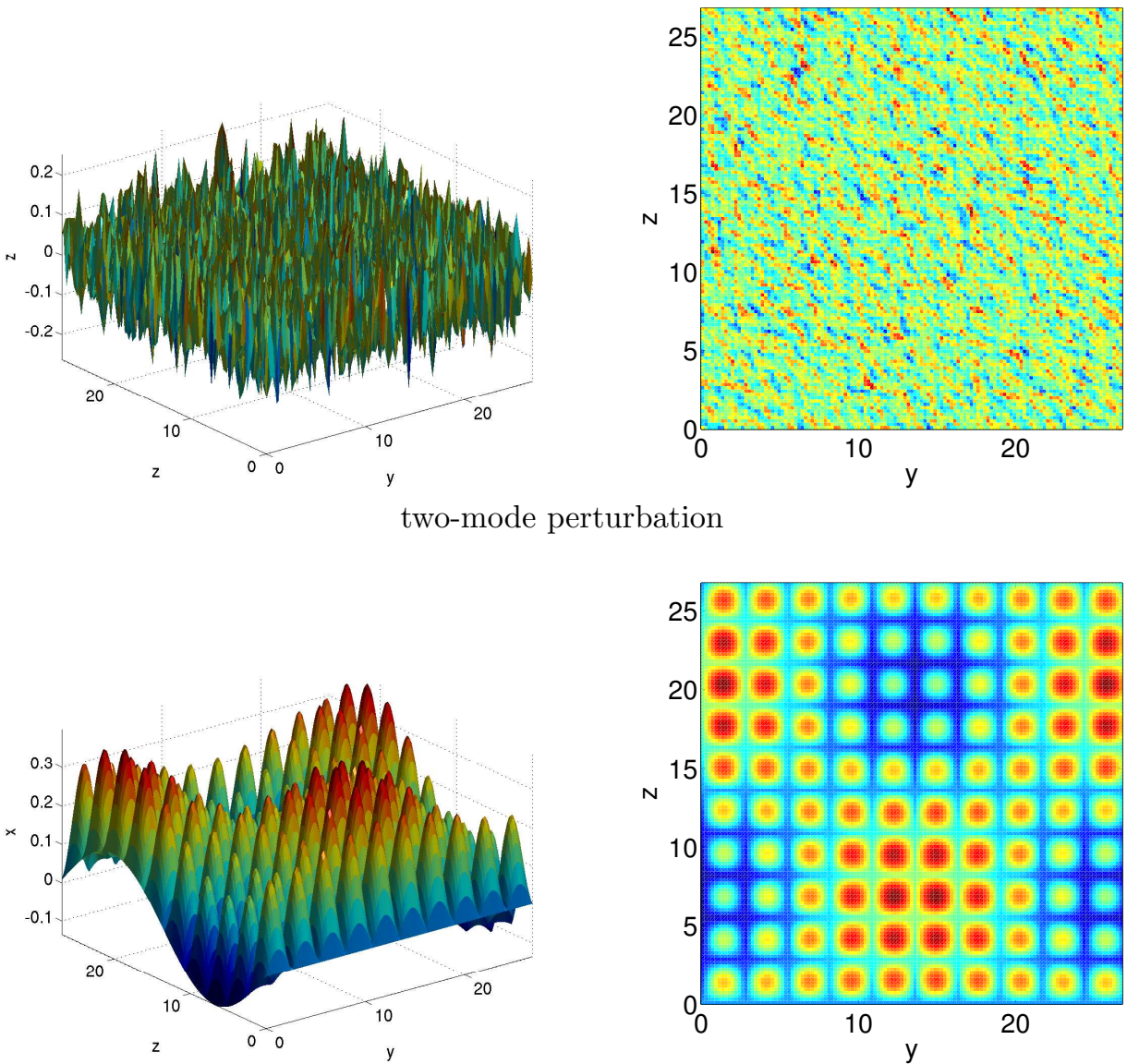

$\eta(y, z)$
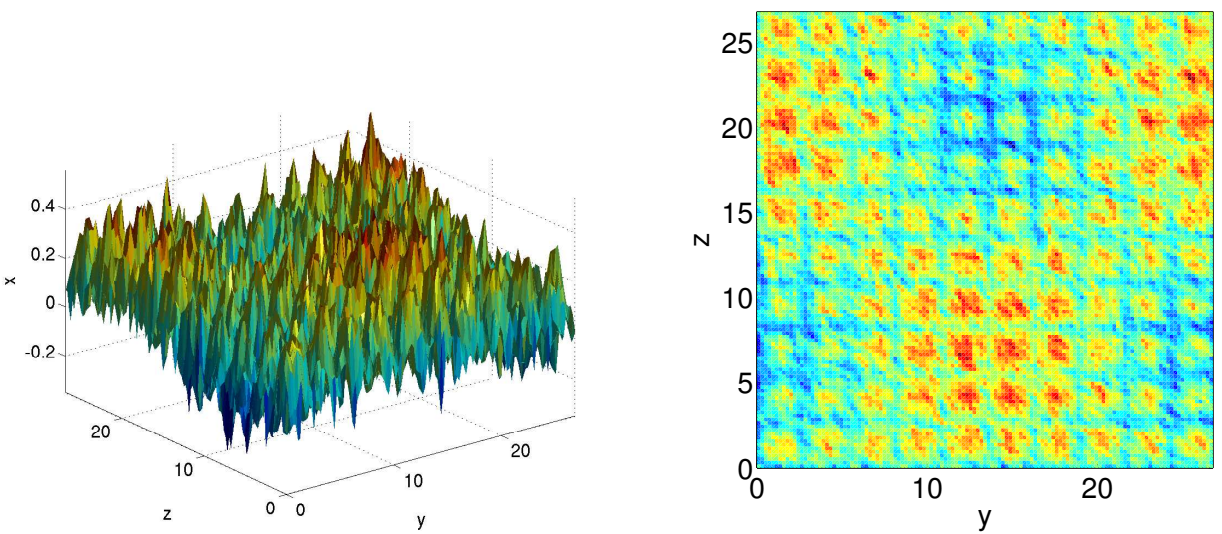

Figure 3: The small-scale stochastic perturbation field $\psi(y, z)$ used to break the symmetry (top row), the initial two-mode perturbation (middle row), andd $\eta(y, z)$ (bottom row). 
defining $k$. For each entry compute the corresponding value of $\widehat{\psi}(k)$ according to (10). Multiply the result by a random phase $e^{i \theta} \cos \phi$, where $\phi$ and $\theta$ are random numbers. The resulting value is used to set $\widehat{\psi}\left(k_{y}, k_{z}\right)$. Take the inverse two-dimensional Fourier transform to obtain $\psi(y, z)$. Finally, $\psi(y, z)$ is normalized so that

$$
\psi(y, z)=\frac{a}{20} \frac{\psi(y, z)}{\psi_{r m s}},
$$

corresponding to a $5 \% \mathrm{rms}$ amplitude for the noise with $\psi_{r m s}=\sqrt{\int_{0}^{k} E_{\psi}(k) \mathrm{d} k}$. Figure 3 shows the noise $\psi(y, z)$, the two-mode perturbation [i.e., $\eta(y, z)$ with $\psi(y, z)=0$ ], and the two-mode perturbation $\eta(y, z)$ with the superposed noise $\psi(y, z)$.

\subsection{Visualization of the instability growth and mixing layer evolution}

The instability evolution is visualized using isosurfaces of the mass fraction of the heavier $\mathrm{SF}_{6}$ gas, evaluated at $m_{S F_{6}}=1 / 2$. The isosurface representation qualitatively captures the center of the mixing layer and clearly shows the growth and interaction of bubble- and spike-like structures. The isosurfaces obtained from the ninth-order WENO simulation at different times as the instability develops are shown in Figs. 4-6 during the early-time evolution, evolution through the reshock, and at later times up to $10 \mathrm{~ms}$. The two sides of the interface - the air side on which the spikes develop and the $\mathrm{SF}_{6}$ side on which the bubbles develop - are also shown.

The isosurfaces at $t=0 \mathrm{~ms}$ in Fig. 4 show the initial perturbed interface on the air and $\mathrm{SF}_{6}$ sides. The large-wavelength perturbation representing the mesh distortion with its small amplitude can only be observed indirectly through its effects on the smallscale perturbation. Following the passage of the incident shock, spikes and bubbles form at $t=0.25 \mathrm{~ms}$. The spikes begin to roll-up, which can barely be seen through $t=3$ ms. At the earliest times shown, the structures grow nearly independently, with little interaction. Mode couplings become significant after $1 \mathrm{~ms}$, with a complex crenulated interface developing just before reshock at $3 \mathrm{~ms}$. Reshock occurs at $t \approx 3.25 \mathrm{~ms}$ (in good agreement with the time of reshock in the experiment) and the bubbles are compressed. Following reshock, inversion occurs with bubbles rapidly transforming into spikes and vice versa. At $t \approx 5 \mathrm{~ms}$, the reflected rarefaction wave induced by the refraction of the shock at the interface, interacts with the evolving interface and stretches the mixing layer in the direction of the $\mathrm{SF}_{6}$, as seen from the location of the interface on the $\mathrm{SF}_{6}$ side. The arrival of the reflected rarefaction represents the last important wave-interface interaction, as the reflected compression wave is very weak. Consequently, the flow nearly becomes a purely decaying flow after $t=6 \mathrm{~ms}$. At $t=10 \mathrm{~ms}$, the flow exhibits a very complex structure. However, remnants of the large bubble, and spike resulting from the large wavelength perturbation are still present. In addition, despite the complex spatial structure, the flow preserves a large degree of symmetry in the largest scales. For times greater than $3.5 \mathrm{~ms}$, the structures grow in amplitude much more rapidly than before the reshock.

Cross-sections of the density in the $(x, z)$-plane centered on the point $x=x_{m i d}$ are also presented at the same evolution times as the mass fraction isosurfaces in Fig. 7, allowing a 'side-on' visualization of the small-scale structures present in the evolving 

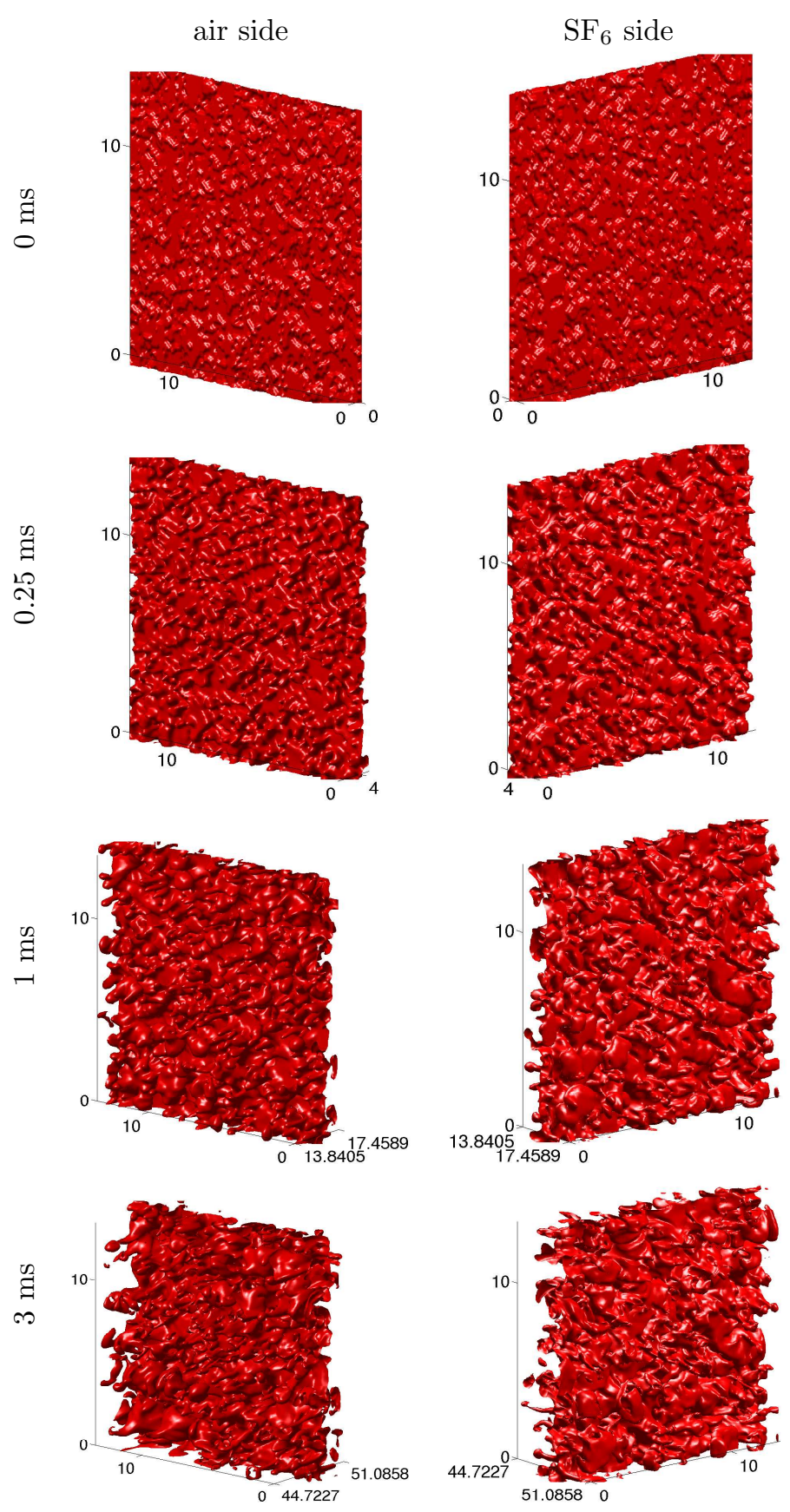

Figure 4: Time-evolution of the mass fraction isosurface $m_{S F_{6}}=1 / 2$ in the multi-mode RichtmyerMeshkov instability for times $t=0,0.25,1$, and $3 \mathrm{~ms}$. Both the air and $\mathrm{SF}_{6}$ sides of the isosurfaces are shown. 

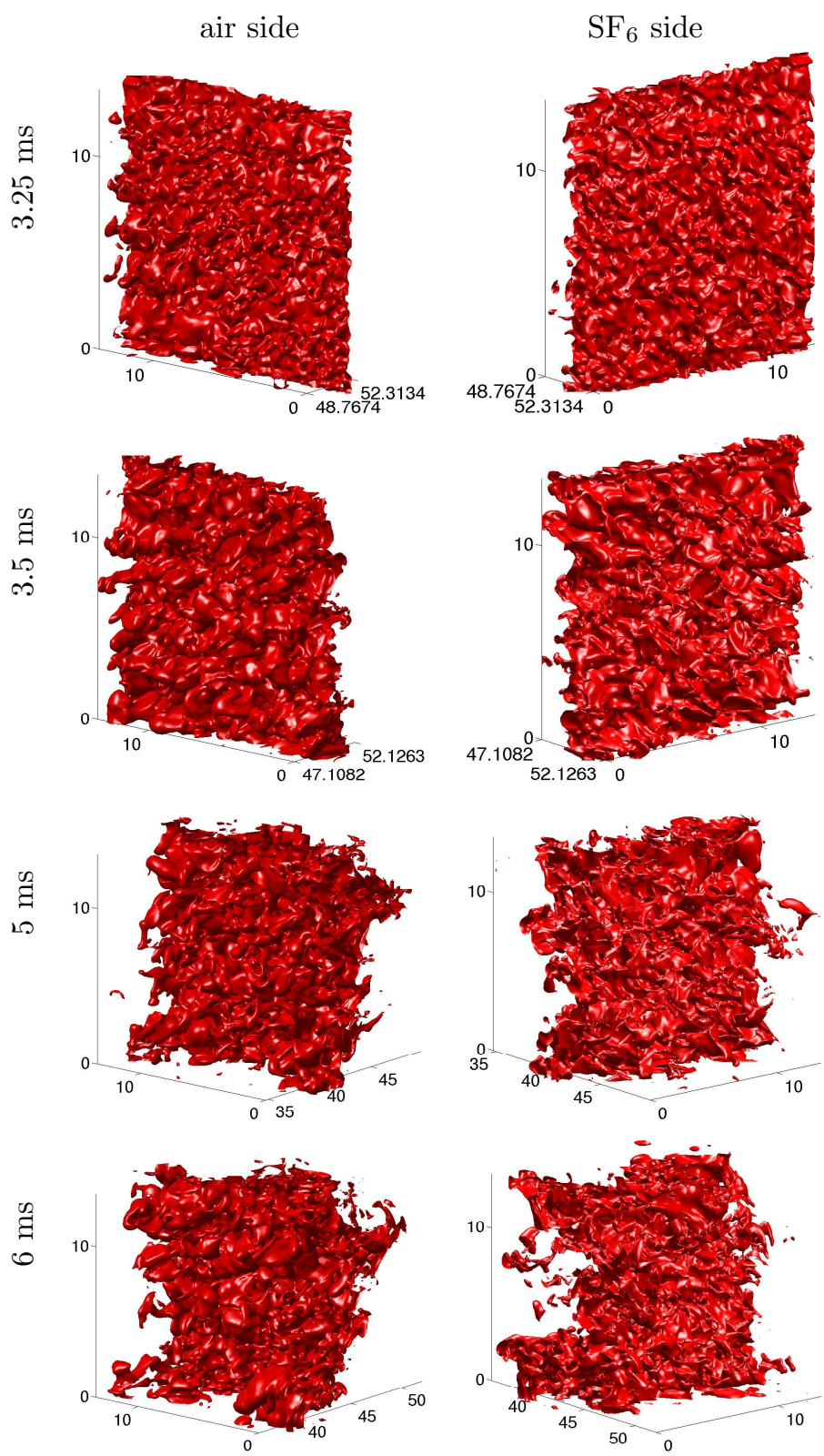

Figure 5: Time-evolution of the mass fraction isosurface $m_{S F_{6}}=1 / 2$ in the multi-mode RichtmyerMeshkov instability for times $t=3.25,3.5,5$, and $6 \mathrm{~ms}$. Both the air and $\mathrm{SF}_{6}$ sides of the isosurfaces are shown. 

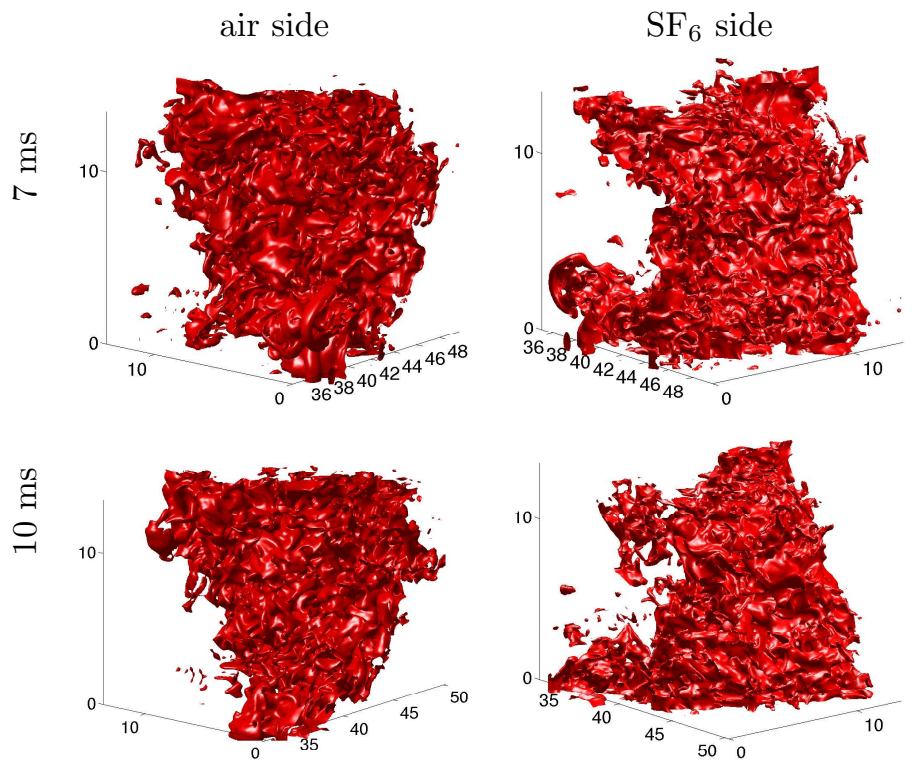

Figure 6: Time-evolution of the mass fraction isosurface $m_{S F_{6}}=1 / 2$ in the multi-mode RichtmyerMeshkov instability for times $t=7$ and $10 \mathrm{~ms}$. Both the air and $\mathrm{SF}_{6}$ sides of the isosurfaces are shown.

mixing layer. The midpoint of the interface is

$$
x_{m i d}(t)=\frac{h_{b}(t)+h_{s}(t)}{2},
$$

which is used to recenter the mixing layer width such that

$$
-\frac{1}{2} \leq \frac{x-x_{m i d}(t)}{h(t)} \leq \frac{1}{2}
$$

Blue and red represent 'pure' fluid and intermediate colors represent 'mixed' fluid. The 'mixing' is solely due to numerical effects, rather than by molecular processes physically present in the experiment. The numerical determination of the bubble and spike front locations $h_{b}$ and $h_{s}$, respectively, is discussed further in Section 5. The compression of the layer at the time of reshock is also clearly seen, followed by a rapid spatial expansion of the mixing layer. The density cross-sections illustrate the development of a well-mixed complex layer at times $t>5 \mathrm{~ms}$.

\subsection{Instability dynamics and the effects of reshock}

The instability dynamics is further visualized using the enstrophy isosurfaces (evaluated at the average value of the enstrophy) on the air (spike) side in Fig. 8. The mass fraction isosurfaces and corresponding density cross-sections are also shown for reference. Reshock occurs at $t \approx 3.25 \mathrm{~ms}$ and the reflected rarefaction wave arrives at $t \approx 5 \mathrm{~ms}$. 

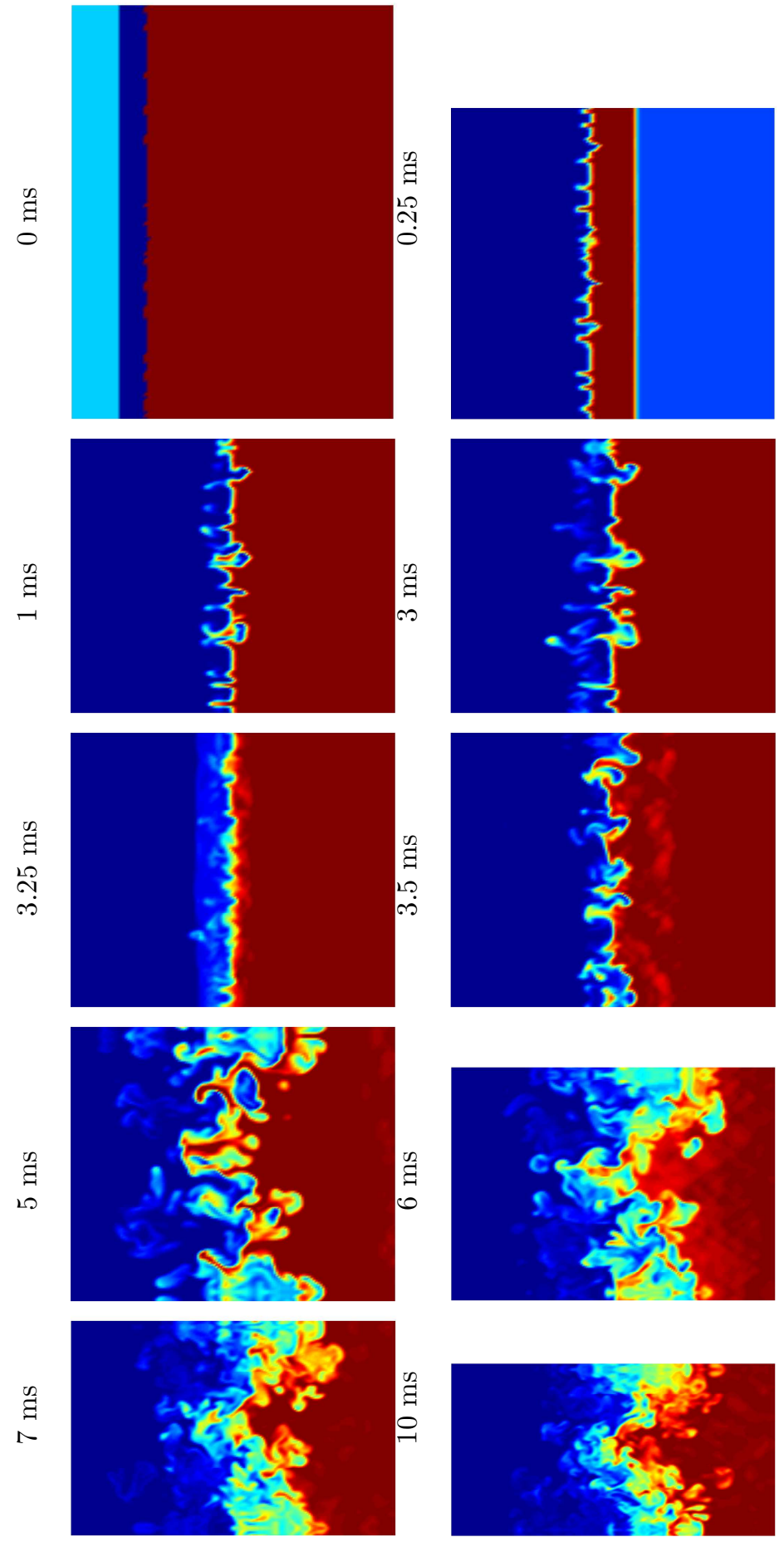

Figure 7: Time-evolution of the density in the multi-mode Richtmyer-Meshkov instability at times $t=0$, $0.25,1,3,3.25,3.5,5,6,7$, and $10 \mathrm{~ms}$. The density cross-sections are shown on the $(x, z)$-plane. Blue represents air and red represents $\mathrm{SF}_{6}$. The height of each image is $27 \mathrm{~cm}$. 

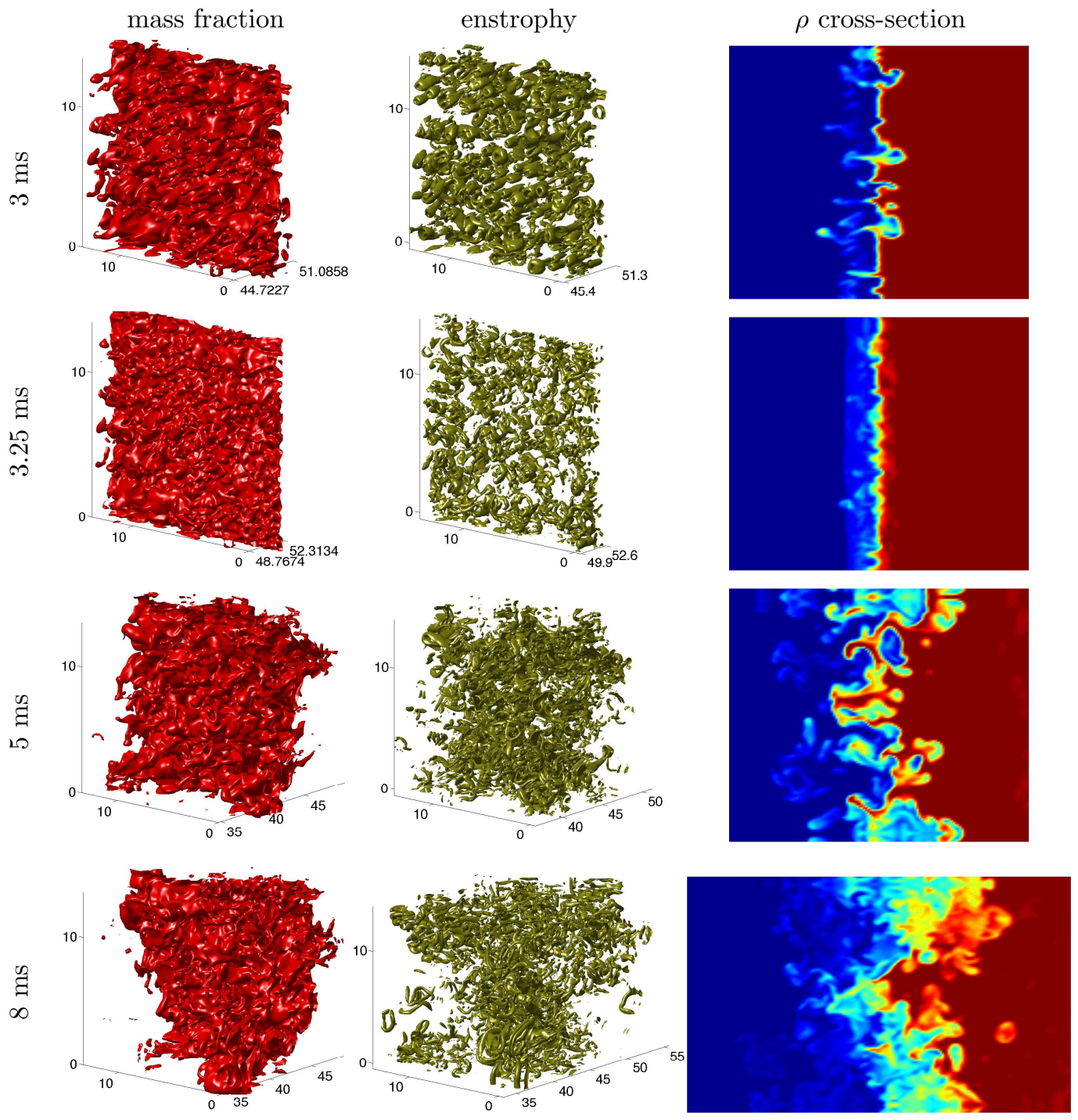

Figure 8: Visualization of the mass fraction isosurface (left column), enstrophy isosurface (middle column), and density $(x, z)$-cross-section (right column), where blue represents air and red represents $\mathrm{SF}_{6}$ at $3,3.25,5$, and $8 \mathrm{~ms}$. The height of each density cross-section is $27 \mathrm{~cm}$. 
Following reshock, the instability develops complex structure and the enstrophy field transforms from elongated tubular structures into short, disordered tubular structures with random orientations. A comprehensive discussion of reshocked two-dimensional Richtmyer-Meshkov instability, including visualizations of density and vorticity fields, mixing layer growth, time evolutions of a broad range of statistics and energy spectra, and the effects of order of WENO reconstruction and grid resolution was previously presented $[14,15,24]$.

\section{Comparison of the Mixing Layer Width with Experimental Data and with the Mikaelian Reshock Model}

The time-evolution of the mixing layer width is of principal interest in experimental, numerical, and modeling studies of the Richtmyer-Meshkov instability. While progress has been made in fielding new experimental diagnostics in studies of continuously- or impulsively-accelerated two-fluid flows, most experimental studies are still limited to visualization of the progression of the instability and inferred perturbation amplitude or mixing layer growth in single- and multimode studies, respectively.

\subsection{Definition of the mixing layer width}

To determine the mixing layer width consider the mole fraction

$$
X(\boldsymbol{x}, t)=\frac{m(\boldsymbol{x}, t) M_{1}}{[1-m(\boldsymbol{x}, t)] M_{2}+m(\boldsymbol{x}, t) M_{1}},
$$

where $m$ is the mass fraction of $\mathrm{SF}_{6}$ and $M_{1}$ and $M_{2}$ are the molecular weights of air and $\mathrm{SF}_{6}$, respectively. Spatially-averaging $X$ in the periodic $y$ - and $z$-directions gives the mean mole fraction along the shock propagation direction

$$
\bar{X}(x, t)=\frac{1}{L_{y} L_{z}} \int_{0}^{L_{z}} \int_{0}^{L_{y}} X(\boldsymbol{x}, t) \mathrm{d} y \mathrm{~d} z,
$$

where $L_{y}$ and $L_{z}$ are the cross-sectional widths of the domain. The spike and bubble front locations, $h_{s}(t)$ and $h_{b}(t)$, are defined as the $x$ position where $\bar{X} \geq \epsilon$ and $\bar{X} \leq 1-\epsilon$, respectively, with $\epsilon=0.01$ used in the present investigation (corresponding to a $1-99 \%$ criterion in the mean mole fraction). The total mixing layer width is then the difference between the bubble and spike front locations,

$$
h(t)=h_{b}(t)-h_{s}(t) .
$$

The same definitions were used in a previous comprehensive two-dimensional study of the Collins and Jacobs [6] single-mode Mach 1.2 air(acetone)/ $\mathrm{SF}_{6}$ experiment using the WENO method $[14,15,24]$. Note that this definition of the mixing layer width is quite sensitive to the choice of $\epsilon$.

The $x$ - $t$ diagram showing the shock and positions of the bubble and spike fronts is shown in Fig. 9. The initial shock reflects at the end wall at $t \approx 2.2 \mathrm{~ms}$ and the reflected shock interacts with the interface at $t \approx 3.25 \mathrm{~ms}$. The reflected shock reaches $x=0$ at $t \approx 4.5 \mathrm{~ms}$ and exits the domain. Before and after reshock, the growth of the bubble and spike fronts is nearly symmetric at this intermediate Atwood number. 


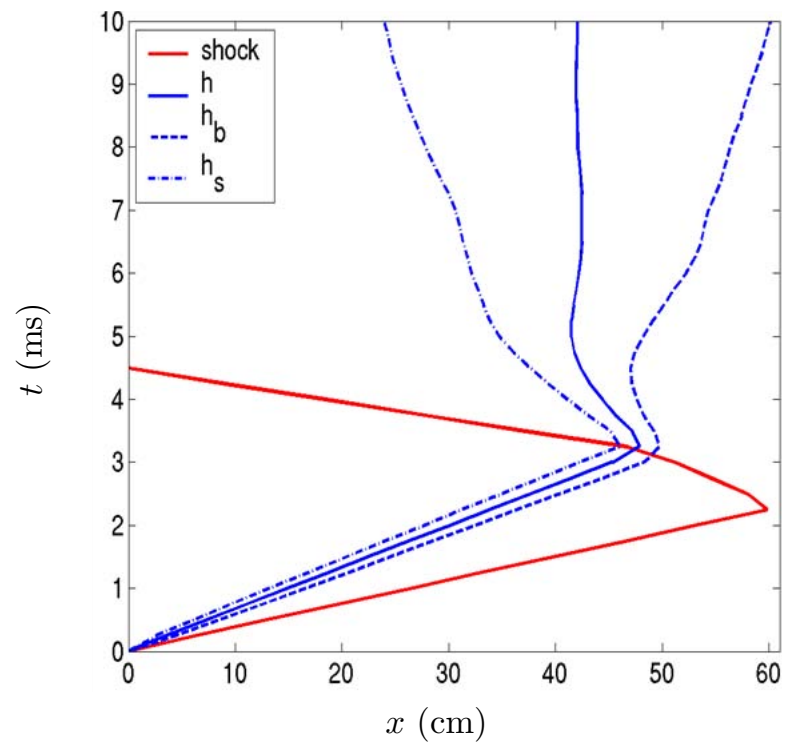

Figure 9: The $x$ - $t$ diagram showing the midpoint of the interface $x_{\text {mid }}-,--$ bubble front location $h_{b}$ - . spike front location $h_{s}$, and shock - . The horizontal distance between the spike and bubble fronts is the mixing layer width $h$.

\subsection{Evolution of the mixing layer width and comparison with the Mikaelian model}

The mixing layer width from the simulation, $h(t)$, is shown in Fig. 10. Table 2 gives a comparison of various pre- and post-reshock quantities obtained from the simulation with the experimental data. The growth rates $\mathrm{d} h / \mathrm{d} t$ agree well with the experimental measurements of $4000 \mathrm{~cm} / \mathrm{s}$ (before reshock) and $32600 \mathrm{~cm} / \mathrm{s}$ (after reshock) [27]: the simulation data is in good agreement with the velocity jumps due to the initial shock passage and to reshock. The mixing layer width also quantitatively agrees with the experimental data after reshock, but overpredicts the data before reshock. The overestimation of the width before reshock is likely related to limited spatial resolution along the shock propagation direction and the choice of initial perturbation (as well as possible membrane break-up effects present in the experiment but not represented in the simulations). As in the simulation of Hill et al. [11], the simulation width prior to reshock was approximately two times larger than the experimentally measured width (as the grid resolution required initial perturbations much larger than those inferred from the experiment). Smaller values of $a$ (requiring much higher spatial resolutions) may provide better quantitative agreement with the pre-reshock amplitude data.

The post-reshock mixing layer widths were also compared to the prediction of the Mikaelian [19] model (2) in Fig. 10, which predicts the velocity

$$
v_{0}=\frac{\mathrm{d} h}{\mathrm{~d} t}=0.28 \Delta u_{1} A^{+} .
$$

The simulation predicts a velocity $v_{0}$ that is $\approx 10 \%$ smaller than the data before reshock and $\approx 3 \%$ smaller than the data after reshock. For a short time following reshock (and 


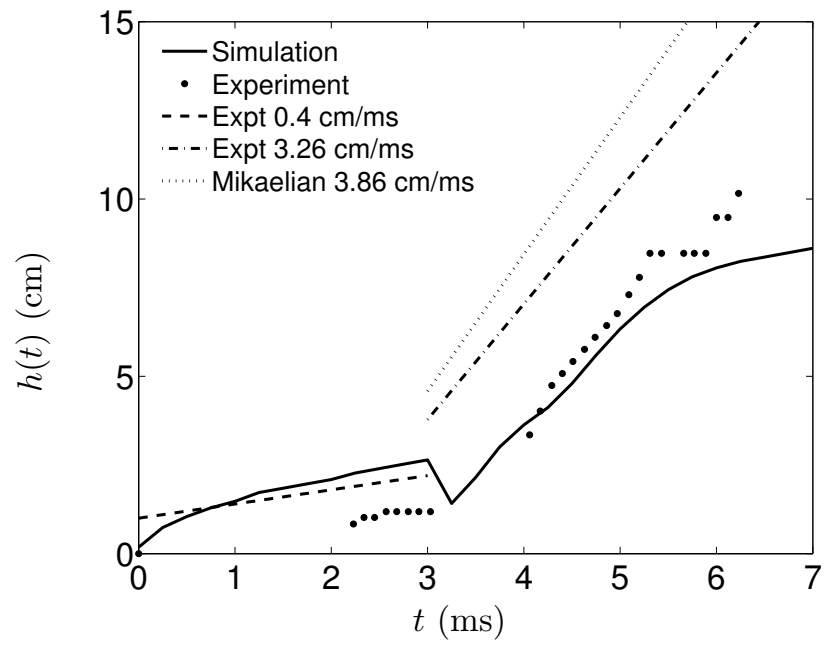

Figure 10: Comparison of the mixing layer width from the simulation with the Vetter-Sturtevant experimental growth rates and data points [27], and with the prediction of the Mikaelian reshock model (2). The simulation had $a=0.0675 \mathrm{~cm}$ and $b=0.00675 \mathrm{~cm}$.

\begin{tabular}{|c||c|c||c|c||}
\hline \multicolumn{1}{|c||}{} & \multicolumn{2}{c||}{ Experimental data } & \multicolumn{2}{c||}{ Simulation data } \\
\hline \hline & Pre-reshock & Post-reshock & Pre-reshock & Post-reshock \\
\hline$\Delta u(\mathrm{~cm} / \mathrm{s})$ & 15000 & 203061 & 15504 & 20584 \\
$A^{+}$ & 0.731 & 0.7 & 0.6352 & 0.6691 \\
$v_{0}(\mathrm{~cm} / \mathrm{ms})$ & 3.07 & 3.98 & 2.76 & 3.86 \\
\hline
\end{tabular}

Table 2: Pre- and post-reshock values of the velocity jump at the interface $\Delta u$, Atwood number $A^{+}$, and mixing layer growth rates $v_{0}$ obtained in the simulation and comparison to experimental data. 


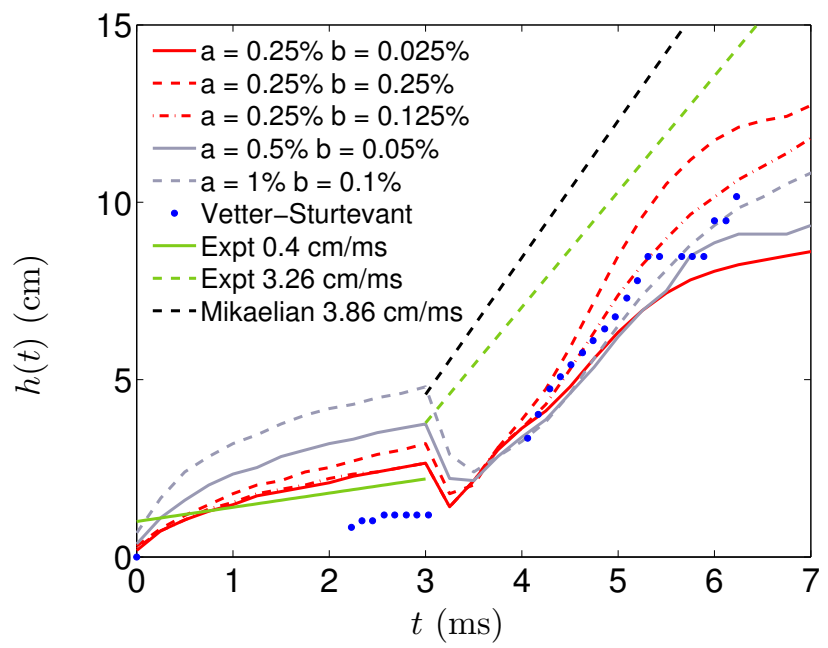

Figure 11: Comparison of the mixing layer width from the simulations using different perturbation amplitudes $a$ and $b$ with the Vetter-Sturtevant experimental growth rates and data points [27], and with the prediction of the Mikaelian reshock model (2).

before the arrival of the reflected rarefaction at $t \approx 5 \mathrm{~ms}$ ), the Mikaelian model predicts a growth rate in generally good agreement with the simulation. Linear post-reshock growth was also recently confirmed in the air $/ \mathrm{SF}_{6}$ experiments of Leinov et al. [16, 17].

\subsection{Sensitivity of the mixing layer width to the initial amplitudes}

The sensitivity of the mixing layer width in the reshocked multimode RichtmyerMeshkov instability has thus far received little attention. As there is freedom in specifying the initial amplitudes in the two-mode perturbation, it is useful to investigate this sensitivity in simulations of the Vetter-Sturtevant experiment. Figure 11 shows the mixing layer widths from a set of simulations with five different sets of values of the perturbation amplitudes $a$ and $b$, with $a$ ranging from $0.25 \%$ to $1 \%$ and $b$ ranging from $0.025 \%$ to $0.25 \%$. As in Section 4 , the percentage refers to the percentage of $L_{z}$. Evidently, the mixing layer width is quite sensitive to the initial amplitudes following the initial shock and before reshock. For nearly all choices of $a$ and $b$, the simulation growth rates agree well with the data before and after reshock. In general, the post-reshock width is less sensitive to the choices of $a$ and $b$. All widths agree quantititatively for approximately $1 \mathrm{~ms}$ following reshock and then begin to differ just before the arrival of the reflected rarefaction at $t \approx 5 \mathrm{~ms}$. Note that larger pre-reshock amplitudes correspond to smaller post-reshock amplitudes. The case corresponding to an $a$ of $0.25 \%$ and $b$ of $0.125 \%$ is in best overall agreement with the data following reshock. Just after reshock and prior to the interaction of the reflected rarefaction with the mixing layer, the simulation growth rates are also in good agreement with the prediction of the Mikaelian model (2). 


\section{Amplification of Turbulence by Reshock}

Experimental and numerical simulation studies of the singly- and multiply-shocked Richtmyer-Meshkov instability have largely focused on the evolution of the mixing layer width $h(t)$. Furthermore, most instability growth models can only predict $h$. However, RANS and LES models must also accurately predict the amplification of turbulence by reshock. Here, the amplification is quantified by considering turbulent energy and enstrophy spectra, and the production terms in the turbulent kinetic energy and enstrophy equations.

\subsection{Amplification of energy spectra}

The amplification of the turbulent kinetic energy and turbulent enstrophy spectra resulting from reshock of the mixing layer is investigated here. The energy associated with each Fourier mode $k$ is obtained by averaging over the extent of the mixing layer to obtain the two-dimensional energy spectrum of a general field $\phi$,

$$
E_{\phi \phi}(k, t)=\frac{1}{2 h(t)} \int_{h_{s}(t)}^{h_{b}(t)}|\widehat{\phi}(k, x, t)|^{2} \mathrm{~d} x
$$

where $\widehat{\phi}(k, x, t)=\frac{1}{(2 \pi)^{2}} \int_{-\infty}^{\infty} \int_{-\infty}^{\infty} \phi(x, y, z, t) \exp \left(-i k_{y} y-i k_{z} z\right) \mathrm{d} y \mathrm{~d} z$ is the Fourier coefficient of the function $\phi(\boldsymbol{x}, t)$ periodic in the $y$-and $z$-directions. Figure 12 shows the time evolution of the turbulent kinetic energy and turbulent enstrophy spectra before reshock, after reshock, and at late times over $1 \mathrm{~ms}$ intervals. Each spectrum is amplified at all spatial scales (i.e., wavenumbers) by one to two orders of magnitude by the reshock, which imparts energy into the layer and generates even smaller scale structures (that become even more challenging to resolve on a numerical grid). Velocity fluctuations and their gradients are thus amplified, as reflected in the increased magnitude of the spectra. The spectra continue to decay relatively slowly after reshock and to the late time shown. At $t=9 \mathrm{~ms}$, both spectra are still considerably larger in magnitude at all wavenumbers than before reshock at $t=3 \mathrm{~ms}$. Fiducials are shown for $k^{-5 / 3}$ and $k^{1 / 3}$ for the turbulent kinetic energy and turbulent enstrophy spectra, respectively. The limited spatial resolution and absence of an explicit subgrid-scale model lead to very short apparent inertial subrange scalings over less than a decade in wavenumber. It is possible that Kolmogorov inertial subranges scalings in three-dimensional homogeneous turbulence, $E(k) \propto k^{-5 / 3}$ and $\Omega(k) \propto k^{2} E(k) \propto k^{1 / 3}$, emerge at even higher spatial resolutions or at greater evolution times. Note that the late-time phase is a 'quasi-decay' phase in which the influence of the shock on the flow field diminishes as time evolves and the fields decay.

\subsection{Amplification of principal turbulence production terms}

The effects of reshock on the principal physical transport processes can also be investigated by considering the production terms in the enstrophy $\left(\Omega=\omega^{2} / 2\right)$ and turbulent kinetic energy $\left(\widetilde{E^{\prime \prime}}=\widetilde{u_{j}^{\prime \prime} u_{j}^{\prime \prime}} / 2\right)$ transport equations

$$
\rho \frac{\mathrm{d} \Omega}{\mathrm{d} t}=P_{\Omega}+S_{\Omega}+C_{\Omega}, \bar{\rho} \frac{\mathrm{d} \widetilde{E^{\prime \prime}}}{\mathrm{d} t}=P_{b}+P_{s}+\Pi+T,
$$




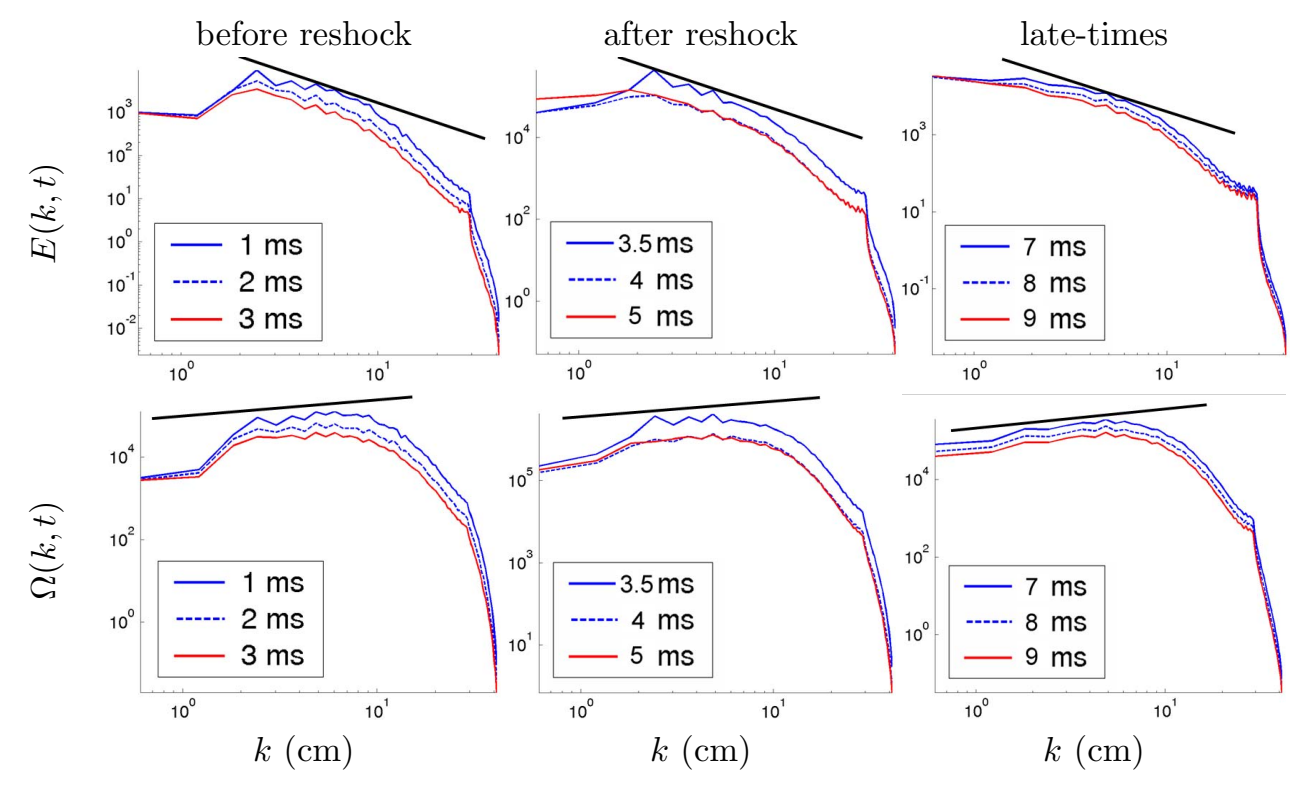

Figure 12: Time-evolution of the turbulent kinetic energy spectrum (top row) and of the turbulent enstrophy spectrum (bottom row). The left, middle, and right columns show the spectra on a log-log scale before reshock, after reshock, and at late times, respectively. A $k^{-5 / 3}$ and $k^{1 / 3}$ fiducial is shown for the turbulent kinetic energy and turbulent enstrophy spectra, respectively.

respectively (note that the enstrophy equation is not averaged). The baroclinic production, stretching, and compression terms in the enstrophy equation are

$$
P_{\Omega} \equiv \frac{\boldsymbol{\omega} \cdot(\boldsymbol{\nabla} \rho \times \boldsymbol{\nabla} p)}{\rho}, S_{\Omega} \equiv \rho \boldsymbol{\omega} \cdot(\boldsymbol{\omega} \cdot \nabla \boldsymbol{u}), C_{\Omega} \equiv-2 \rho \Omega \boldsymbol{\nabla} \cdot \boldsymbol{u}
$$

respectively, and the buoyancy production, shear (Reynolds stress) production, pressuredilatation, and transport terms in the turbulent kinetic energy equation are

$$
P_{b} \equiv-\overline{u_{j}^{\prime \prime}} \frac{\partial \bar{p}}{\partial x_{j}}, P_{s} \equiv-\overline{\rho u_{i}^{\prime \prime} u_{j}^{\prime \prime}} \frac{\partial \widetilde{u}_{i}}{\partial x_{j}}, \Pi \equiv \overline{p^{\prime} \frac{\partial u_{j}^{\prime \prime}}{\partial x_{j}}}, T \equiv-\frac{\partial}{\partial x_{j}}\left(\overline{\rho E^{\prime \prime} u_{j}^{\prime \prime}}+\overline{p^{\prime} u_{j}^{\prime \prime}}\right),
$$

respectively. Here, the Reynolds and Favre averages, $\bar{\phi}$ and $\widetilde{\phi}$, are defined as the spatial averages over the periodic directions

$$
\bar{\phi}(x, t)=\frac{1}{L_{x} L_{z}} \int_{0}^{L_{z}} \int_{0}^{L_{y}} \phi(\boldsymbol{x}, t) \mathrm{d} y \mathrm{~d} z, \quad \widetilde{\phi}(x, t)=\frac{\overline{\rho \phi}}{\bar{\rho}}
$$

and the corresponding Reynolds and Favre fluctuating fields are $\phi(\boldsymbol{x}, t)^{\prime}=\phi(\boldsymbol{x}, t)-\bar{\phi}(x, t)$ and $\phi(\boldsymbol{x}, t)^{\prime \prime}=\phi(\boldsymbol{x}, t)-\widetilde{\phi}(x, t)$, respectively. Note that the transport equations are derived from averaging the Euler (rather than Navier-Stokes) equations, so that (19) do not contain the turbulent kinetic energy and enstrophy dissipation rate terms. In such simulations, it is possible to define a turbulent kinetic energy dissipation rate in terms of the turbulent enstrophy $\widetilde{\Omega^{\prime \prime}}=\widetilde{\omega_{j}^{\prime \prime} \omega_{j}^{\prime \prime}} / 2$ by $\widetilde{\epsilon^{\prime \prime}}=\widetilde{E^{\prime \prime}} \widetilde{\omega^{\prime \prime}} \equiv \widetilde{E^{\prime \prime}} \sqrt{2 \widetilde{\Omega^{\prime \prime}}}$. 

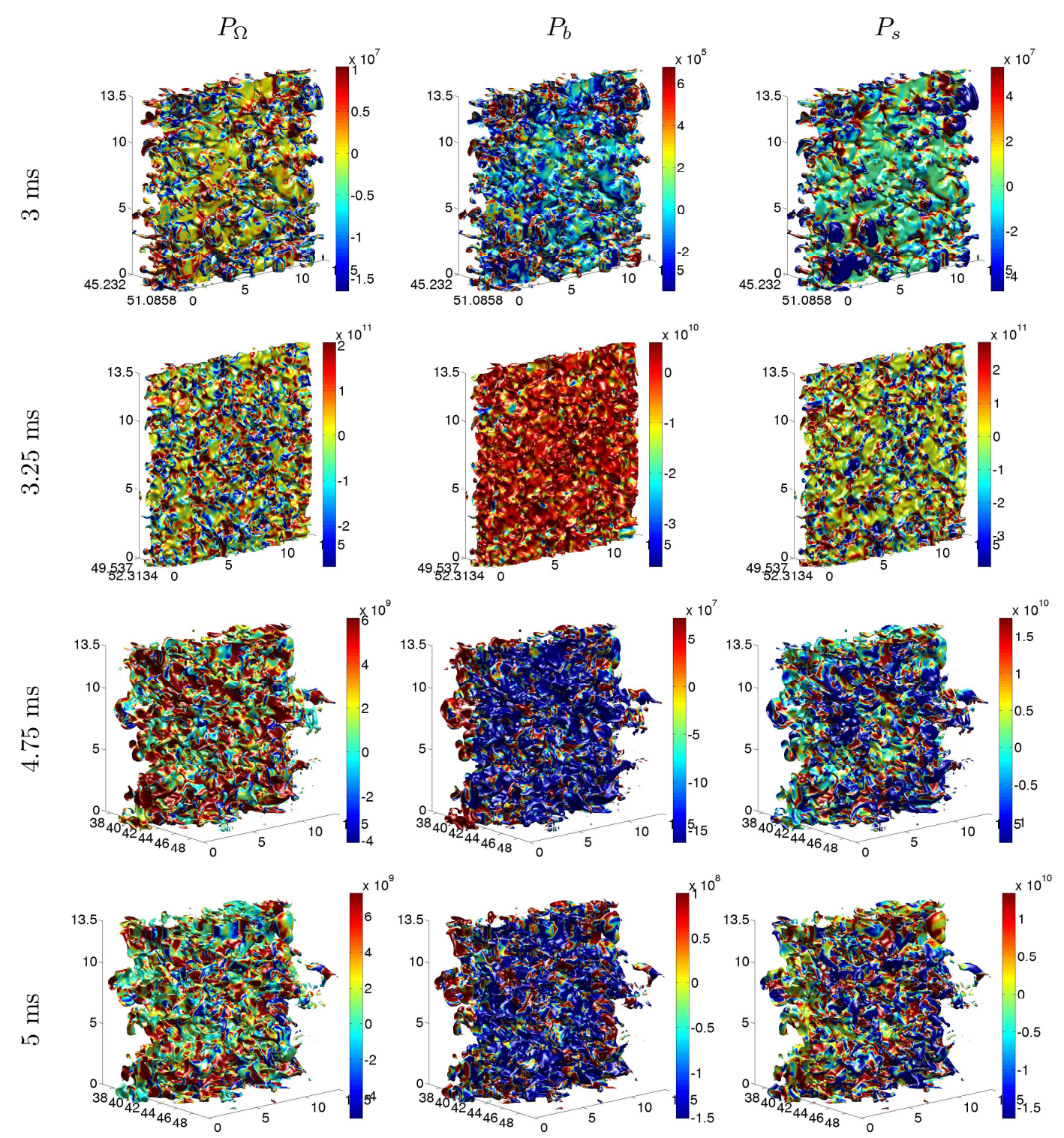

Figure 13: The baroclinic production of enstrophy $P_{\Omega}$, buoyancy production of kinetic energy $P_{b}$, and shear production of kinetic energy $P_{S}$ visualized on the mass fraction isosurface $m_{S F_{6}}=1 / 2$ before reshock $(3 \mathrm{~ms})$, after reshock $(3.25 \mathrm{~ms})$, at $4.75 \mathrm{~ms}$, and at $5 \mathrm{~ms}$ (corresponding to the arrival of the reflected rarefaction at the interface). 
Figure 13 shows the mass fraction isosurface $m_{S F_{6}}=1 / 2$ colored by the baroclinic enstrophy production $P_{\Omega}$, buoyancy production $P_{b}$, and shear production $P_{s}$ at $3,3.25$, 4.75 , and $5 \mathrm{~ms}$. The color scale indicates the magnitude of each term, clearly indicating the amplification of these fields by many orders of magnitude through the reshock of the mixing layer. After reshock, the magnitudes slowly decrease in general. These fields are not currently available from experimental studies of the Richtmyer-Meshkov instability and must be obtained from numerical simulation data. Quantitative estimates of these amplifications by reshock of the evolving mixing layer are essential for developing and validating higher fidelity Reynolds-averaged Navier-Stokes (RANS) models for reshocked Richtmyer-Meshkov instability-induced mixing. Reshock is also necessary to achieve a sufficiently complex mixing layer so that applying traditional turbulent averages of the form (22) is meaningful [24].

\section{Discussion and Conclusions}

A two-mode initial perturbation with random noise was constructed to model the reshocked Mach 1.5 air/ $\mathrm{SF}_{6}$ Vetter-Sturtevant experiment. As in other simulations of the multimode Richtmyer-Meshkov instability [11, 26], random noise was used to break symmetry and to accelerate the development of nonlinearity. A visualization of the instability evolution through the mass fraction and enstrophy isosurfaces, and density crosssections was presented to exhibit the initially linear instability growth, the subsequent nonlinear interactions among structures, and the development of complex flow structure after reshock. The enstrophy isosurface showed a qualitative change after reshock from long, elongated tubes aligned along the direction of shock propagation to small, short tubular structures with random orientations characteristic of turbulent flows.

It was shown that the growth rate of the mixing layer before and after reshock agreed well with the experimentally measured growth rates and with the prediction of the Mikaelian reshock model [19]. The simulation amplitude overpredicted the experimental amplitude before reshock due to the limited spatial resolution and large initial perturbation amplitudes, but was in good agreement with the data after reshock. A parametric study of the mixing layer width growth was also performed for several choices of the inital two-mode perturbation amplitudes $a$ and $b$. The mixing layer width was found to be quite sensitive to the initial amplitudes following the initial shock and before reshock. For nearly all $a$ and $b$, the simulation growth rates agreed well with the data before and after reshock. The post-reshock widths were less sensitive to the choices of $a$ and $b$. All widths agreed quantititatively for approximately $1 \mathrm{~ms}$ following reshock and then began to diverge just before the arrival of the reflected rarefaction at $t \approx 5 \mathrm{~ms}$. The effects of order and resolution on the mixing layer width and other quantities were not considered here due to the computational expense of simulations with resolutions greater than $513 \times 257^{2}$.

While the evolution of the mixing layer width is of central importance to assessing the predictions of RANS and LES models, other dynamics of the layer are more directly related to the properties of turbulence in the mixing layer. For example, turbulence models must accurately predict the amplification of turbulence by the reshock. The amplification of the turbulence intensity in the mixing layer following reshock was quantified here using the turbulent kinetic energy and turbulent enstrophy spectra, both of which increased by one to two orders of magnitude. The effects of reshock were also quantified by 
showing the strong amplification and subsequent slow decay of the baroclinic enstrophy production, buoyancy production, and shear production terms on the $\mathrm{SF}_{6}$ mass fraction one-half isosurface.

The reduced numerical dissipation in formally high-order methods, coupled with consideration of the computational cost, suggests that such methods are well-suited for simulating complex multiscale flows with shocks. Lower dissipation simulations preserve small-scale structures and provide a more complete representation of the flow dynamics. Low-order representations of complex flow physics are considerably different than highorder representations and, in general, a broad range of quantities should be considered to differentiate between the predictions of different numerical methods [24]. The use of formally higher-order methods is also more computationally efficient than increasing the grid resolution, as demonstrated for the two-dimensional Richtmyer-Meshkov instability [14], leading to a significant advantage in multidimensional simulations of such flows. While definitive experimental data corresponding to the quantities considered in the present study is required to assess the simulation predictions, higher order higher resolution simulations will likely provide higher fidelity, detailed data for the forseeable future. Thus, the current study has important implications for developing more predictive turbulent transport models describing complex, shock-driven flows. Hybrid high-order WENO/central difference Navier-Stokes simulations of Richtmyer-Meshkov instability growth that further reduce the effects of numerical dissipation in smooth flow regions are in progress.

\section{Acknowledgment}

This work was performed under the auspices of the U.S. Department of Energy by Lawrence Livermore National Laboratory under Contract DE-AC52-07NA27344. This work was originally presented at the Ninth International Workshop on the Physics of Compressible Turbulent Mixing held 19-23 July, 2004 in Cambridge, United Kindgom and at the Tenth International Workshop on the Physics of Compressible Turbulent Mixing held 17-21 July, 2006 in Paris, France.

[1] Balsara D S, Shu C-W. Monotonicity preserving weighted essentially non-oscillatory schemes with increasingly high order of accuracy. J Comput Phys, 2000, 160: 405-452

[2] Boris J P, Grinstein F F, Oran E S, Kolbe R L. New insights into large eddy simulation. Fluid Dyn Res, 1992, 10: 199-228

[3] Brouillette M. The Richtmyer-Meshkov instability. Annu Rev Fluid Mech, 2002, 34: 445-468

[4] Cohen R H, Dannevik W P, Dimits A M, Eliason D E, Mirin A A, Zhou Y, Porter D H, Woodward P R. Three-dimensional simulation of a Richtmyer-Meshkov instability with a two-scale initial perturbation. Phys Fluids, 2002, 14: 3692-3709

[5] Colella P, Woodward P R. The piecewise parabolic method (PPM) for gas-dynamical simulations. J Comput Phys, 1984, 54: 174-201

[6] Collins B D, Jacobs J W. PLIF flow visualization and measurements of the Richtmyer-Meshkov instability of an air $/ \mathrm{SF}_{6}$ interface. J Fluid Mech, 2002, 464: 113-136

[7] Cook R C, McEachern R L, Stephens R B. Representative surface profile power spectra from capsules used in Nova and Omega implosion experiments. Fusion Tech, 1999, 35: 224-228

[8] Drikakis D. Embedded turbulence model in numerical methods for hyperbolic conservation laws. Int J Numer Methods in Fluids, 2002, 39: 763-781

[9] Drikakis D. Advances in turbulent flow computations using high-resolution methods. Prog Aerosp Sci, 2003, 39: 405-424

[10] Drikakis D, Hahn M, Mosedale A, Thornber B. Large eddy simulation using high-resolution and high-order methods. Phil Trans Royal Soc London A, 2009, 367: 2985-2997 
[11] Hill D J, Pantano C, Pullin D I. Large-eddy simulation and multiscale modelling of a RichtmyerMeshkov instability with reshock. J Fluid Mech, 2006, 557: 29-61

[12] Jameson L. High order schemes for resolving waves: number of points per wavelength. J Sci Comput, 2000, 15: 417-439

[13] Kreiss H O, Oliger J. Comparison of accurate methods for the integration of hyperbolic equations. Tellus, 1972, 24: 199-215

[14] Latini M, Schilling O, Don W S. Effects of WENO flux reconstruction order and spatial resolution on reshocked two-dimensional Richtmyer-Meshkov instability. J Comput Phys, 2007, 221: 805-836

[15] Latini M, Schilling O, Don W S. High-resolution simulations and modeling of reshocked single-mode Richtmyer-Meshkov instability: comparison to experimental data and to amplitude growth model predictions. Phys Fluids, 2007, 19: 024104-1-024104-19

[16] Leinov E, Sadot O, Formoza A, Malamud G, Elbaz Y, Levin L A, Ben-Dor G, Shvarts D. Investigation of the Richtmyer-Meshkov instability under re-shock conditions. Phys Scripta T, 2008, 132 014014-1-014014-5

[17] Leinov E, Malamud G, Elbaz Y, Levin L A, Ben-Dor G, Shvarts D, Sadot O. Experimental and numerical investigation of the Richtmyer-Meshkov instability under re-shock conditions. J Fluid Mech, 2009, 626: 449-475

[18] Meloon M R. Models of Richtmyer-Meshkov instability in continuously stratified fluids [Ph D thesis]. California Institute of Technology, 1998

[19] Mikaelian K O. Turbulent mixing generated by Rayleigh-Taylor and Richtmyer-Meshkov instabilities. Physica D, 1989, 36: 343-357

[20] Quirk J J, Karni S. On the dynamics of a shock-bubble interaction. J Fluid Mech, 1996, 318: $129-163$

[21] Read K I. Experimental investigation of turbulent mixing by Rayleigh-Taylor instability. Physica D, 1984, 12: $45-58$

[22] Roe P L. Approximate Riemann solvers, parameter vectors, and difference schemes. J Comput Phys, 1981, 43: 357-382

[23] Schilling O, Jacobs J W. Richtmyer-Meshkov instability and re-accelerated inhomogeneous flows. Scholarpedia, 2008, 3: 6090

[24] Schilling O, Latini M, Don W S. Physics of reshock and mixing in single-mode Richtmyer-Meshkov instability. Phys Rev E, 2007, 76: 026319-1-026319-28

[25] Shu C-W. High order weighted essentially nonoscillatory schemes for convection dominated problems. SIAM Rev, 2009, 51: 82-126

[26] Ukai S, Genin F, Srinivasan S, Menon S. Large eddy simulation of re-shocked Richtmyer-Meshkov instability. AIAA Paper, 2009, 2009-944

[27] Vetter M, Sturtevant B. Experiments on the Richtmyer-Meshkov instability of an air/SF 6 interface. Shock Waves, 1995, 4: 247-252

[28] von Kármán T. Progress in the statistical theory of turbulence. Proc Nat Acad Sci, 1948, 34: $530-539$

[29] Youngs D L. Numerical simulation of turbulent mixing by Rayleigh-Taylor instability. Physica D, 1984, 12: $32-44$

[30] Zhang Y-T, Shi J, Shu C-W, Zhou Y. Numerical viscosity and resolution of high-order weighted essentially nonoscillatory schemes for compressible flows with high Reynolds numbers. Phys Rev E, 2003, 68: 046709-1-046709-16 